\title{
Fitting the fracture curve of concrete as a density function pertaining to the generalized extreme value family
}

\author{
A.F. Canteli ${ }^{\mathrm{a}}$, L. Castanon-Jano ${ }^{\mathrm{b}}$, H. Cifuentes ${ }^{\mathrm{c}, *}$, M. Muñiz-Calvente $^{\mathrm{a}}$, E. Castillo ${ }^{\mathrm{d}}$ \\ ${ }^{\text {a }}$ Dept. of Construction and Manufacturing Engineering, University of Oviedo, 33203 Gijón, Spain \\ b Dept. of Transport, Projects and Processes Technology, University of Cantabria, 39005 Santander, Spain \\ ${ }^{c}$ Escuela Técnica Superior de Ingeniería, Universidad de Sevilla, 41092 Sevilla, Spain \\ ${ }^{\mathrm{d}}$ Dept. of Applied Mathematics and Computational Sciences, University of Cantabria, 39005 Santander, Spain
}

\section{A R T I C L E I N F O}

\section{Keywords:}

Fracture of quasi-brittle materials

Size-independent specific fracture energy of concrete

Density function

Generalized extreme value family

\begin{abstract}
A B S T R A C T
The load-displacement $(P-\delta)$ curve, recorded during the fracture process of concrete in three-point bending tests, is supposed to depict a fracture phenomenon of statistical character that can be suitably described by a density function pertaining to the generalized extreme value family, which proves to be maximal Fréchet, as a particular case of heavy tail distributions. Since the proposed analytical function fits the test record throughout, the nonmeasured fracture work, corresponding to the upper asymptotic tail of the fracture curve $P-\delta$, is expected to be measured in a more reliable and accurate way than using other methods currently recommended to evaluate the total fracture energy of concrete. The general scale parameter $\Omega$, identified as the area under the fracture curve, and the three parameters of the Fréchet density function are estimated by fitting the recorded data to the experimental $P$ - $\delta$ curve using a specific Matlab program. The model is applied to fit experimental fracture curves from an ample 3-PB test program on notched specimens for different self-compacting concrete mixes. The results obtained for the size-independent specific fracture energy are compared with those provided by other wellestablished conventional approaches. In both instances, the suitability of the proposal is confirmed.
\end{abstract}

\section{G R A P H I C A L A B S T R A C T}

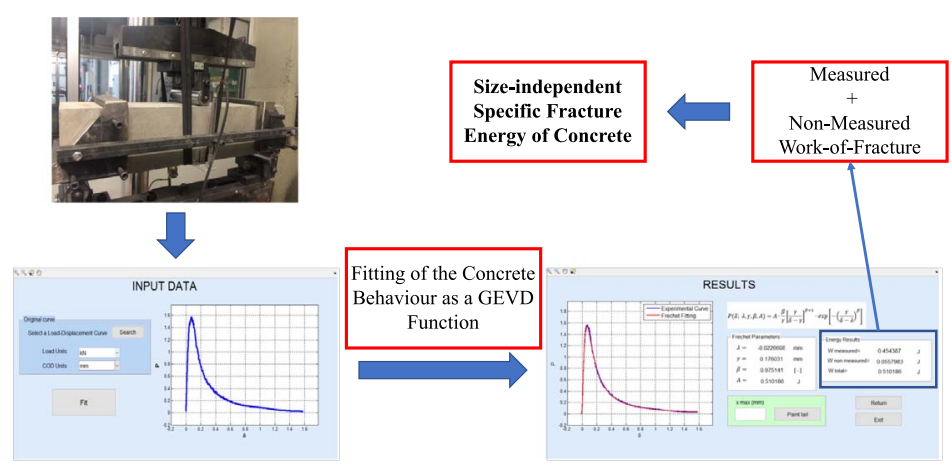

\section{Introduction}

The reliable estimation of the area under the $P-\delta$ (respectively $P$ $C M O D$ ) fracture curve, identified as the total fracture energy dissipated during the fracture process of quasi-brittle materials, in particular of concrete [1], requires suitable and accurate fitting of the test data recorded not only at the lower but the upper or even far upper tail of the fracture curve $[2,3]$. Due to testing limitations, one end point of the

\footnotetext{
* Corresponding author.

E-mail address: bulte@us.es (H. Cifuentes).
} 
test at a certain displacement (or CMOD), known as the test limit, is adopted by interrupting prematurely the test record before complete failure of the specimen occurs. Though the area corresponding to this unattainable terminal phase of the test fracture may appear negligible, it represents a significant part of the total fracture energy that cannot be disregarded because the vast extension of the associated displacement while the low load values are approaching asymptotically to zero. In fact, this non-measured work of fracture is considered for some authors [4-6] as the main cause for the size dependency obtained in the specific fracture energy if 3-PB test is performed as indicated by RILEM work-offracture method [7].

To achieve this goal, two different but complementary fitting steps are generally proposed for measuring the fracture energy. In the first one, the area under the recorded test data is directly measured without a particular analytical expression is needed to fit the fracture curve data. In the second one, exponential or hyperbolic solutions are suggested for the extrapolation of the far upper tail of the fracture curve [4-6] without representing a fully convincing solution to the problem. In any case, the assessment of the fracture energy is not straightforward. First, sudden load jumps and irregularities commonly occur in the post-pick region, due to successive aggregate fractures, impeding the load to be smoothly recorded. Furthermore, the accurateness in the estimation of the marginal energy is influenced by the choice of the initiation point of the extrapolation due to the limiting part of the far upper tail of the fracture curve giving support to the extrapolation [1]. Finally, the questionable suitability of the analytical solution proposed adds uncertainty to the estimation of the marginal energy.

In this paper, an alternative proposal is presented allowing a global and robust analytical fitting of the fracture curve to be achieved. The approach is founded on the presumably statistical character of maxima of the fracture phenomenon observed in the concrete bulk where random local failures progress exhibiting an asymptotic trend. The coarse aggregate structure and its random distribution, inherent to concrete, imply high inhomogeneity of the material evidenced by the peak and post-peak branch of the load-displacement curve of concrete, which explains the statistical nature of the fracture mechanism of concrete as stated by Hu et al. in a recent paper [8]. This suggests the fracture curve shape to be identified as a density function of the generalized extreme value (GEV) family for maxima.

In this work, a novel proposal is made for optimal fitting of the whole fracture curve of concrete from the recorded test results, along both the pre- and post-pick regions, based on an analytical solution pertaining to the GEV distribution. In this way, the asymptotic far upper tail of the fracture curve is also included in the proposal without lack of continuity with the rest of the curve.

\section{Fitting the fracture curve as a density function of the generalized extreme value (GEV) family}

In brittle materials, failing of the first local element failure incites, as a chain reaction, a global component failure so that the weakest link principle is applicable. Other than this family, quasi-brittle materials, as plain concrete, exhibit a steady local failure process with ubiquitous progress so that generalized microcracking propagation extends throughout until the accepted failure. This kind of fracture suggests a certain statistical law underlying the microcracking extension in the 3PB test, which fits the fracture curve of concrete. The latter is identified as the load-displacement (respectively, load-CMOD) curve according to a law of maxima referred to the stronger links, which gradually give up their integrity during the failure process in an asymptotic way thus justifying a heavy tail distribution. In this way, possible discontinuities or inconsistencies by the recorded data arising during the test, as for instance jumps due to aggregate failures, are smoothed.

In order to avoid a correction of the work of fracture to achieve a size-independent value of the specific fracture energy of concrete, an "ad-hoc" fixture was proposed, see [4-6]. This implies a premature test ending, in case that self-weight compensation is provided, whereas the key point consists in how to estimate, through extrapolation of the fracture curve, the remaining work of fracture to be expected beyond such a test limit [9-11].

Different exponential or hyperbolic shaped functions are currently applied to fit the far upper tail of the fracture curve for concrete specimens by extrapolation [1,4-6]. From a physical or functional perspective such a procedure is considered not entirely satisfactory since this fitting is only supported by the recorded results of an arbitrarily selected after-peak region of the fracture curve, namely those showing a positive curvature pertaining to the last branch of the curve approaching to the abscissa axis, i.e. the tail of the curve. Contrary, data results preceding the maximum load value as well as the immediate part of the after peak region of the $P-\delta$ curve are disregarded in this fitting process though both, notably, represent regions not suitable to be fitted by the arbitrary selected curve. Moreover, the transition point between this local fitting and the initial part of the $P$ - $\delta$ curve is discretionary chosen thus conditioning the boundary conditions of the curve searched [1]. In this way, the extrapolation to very large $\delta$ values, implying the fitted curve outside the test scope, seems to be unreliable and as such, questionable.

As an alternative, the whole load-displacement curve $P-\delta$ by quasibrittle materials is supposed to depict a fracture process of statistical character, due to the random successive local failures succeeding by the huge number of material units involved by the cement past and aggregates. Such a process may be adequately represented by a generalized extreme value (GEV) distribution [12], which represents a particular case of heavy tail distributions [13-15]. This family of distributions is occasionally applied for prediction of natural disasters of any kind and is characterized by exhibiting a tail heavier than that provided by the exponential distribution. With such a solution, not only the upper tail but the whole $P-\delta$ curve is fitted. The resemblance between the pdfs of such distributions, which evidence an asymptotic far upper tail, and the concrete fracture energy curves resulting from experimental tests supports "a priori" the assumption of such a probabilistic background inherent to the failure process.

The unknown heavy tailed distribution, theoretically suitable to be assumed, can be identified asymptotically with a distribution pertaining to the generalized extreme value (GEV) family [12]. The suitable particular distribution implied, from which only the Fréchet, Gumbel or Weibull distributions are possible candidates, according to the extreme value statistics, will be decided from the outgoing fitting parameters of the GEV distribution. In fact, from the point of view of a valid range condition, only the Fréchet for maxima and the Weibull for minima are suitable solutions to reproduce the $P-\delta$ fracture curve. This is because the characteristics of the recorded results along the fracture process implying limited lower value and apparently unlimited upper value of the displacement. Intuitively, maxima values of the displacement, or alternatively crack mouth opening displacements (CMOD), are the expected reference magnitudes to be considered, thus requiring an extreme value distribution family for maxima, while load, after previous normalization by the fracture work, may be related to probability. Accordingly, the Weibull option should be discarded, and a Fréchet distribution for maxima is the only possible fitting solution for the $P-\delta$ fracture curve. In any case, such theoretical considerations should be confirmed through the application to the real test $P-\delta$, respective $P$-CMOD registers.

The following advantages are expected using the Fréchet distribution for fitting the fracture curve:

- An extreme value model is supposed to describe statistically the physical fracture phenomenon occurring during the test as the relation existing between the increasing displacement and the locally varying external forces necessary to overcome the cohesion in the process zone.

- The heavy tailed distribution proposed belongs to a threeparameter Fréchet distribution, thus profiting of the properties exhibited by such distributions as representing the only asymptotic solution 

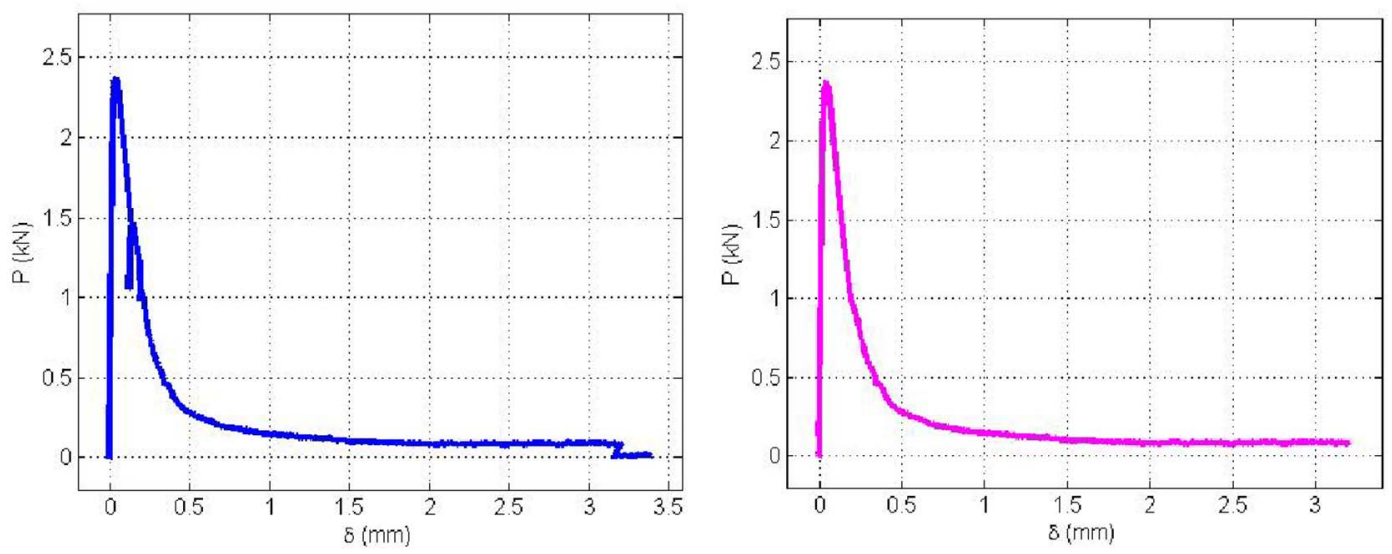

Fig. 1. Example of fracture curve of a concrete as recorded directly from test (left) and after filtering (right).

whichever the fracture curve could be.

- The assumed existence of an underlying statistical model contributes to a more reliable parameter estimation avoiding the arbitrariness of adopting different kinds of regression solutions as a function of the particular material tested. Possibly, physical interpretation can be assigned to the model parameters.

- Since all experimental recorded data from both the pre- and postpick regions are included in the assessment, an extrapolation of the $P-\delta$ curve beyond the test limit to assess the non-measured fracture energy may be objectively justified for fitting the asymptotic upper tail with enhanced reliability.

- The model ensures continuity and smoothness of the fracture curve even if irregular jumps are recorded or even partial lack of records arise due to successive failures of the brittle aggregates.

- The analytical expression obtained for the $P-\delta$ curve and the physical meaning of the intervening parameters entail considerable advantages when analyzing the characteristics and trends of the experimental results obtained for different materials or test conditions, in particular, in the scatter analysis.

- An "ad-hoc" Matlab program, developed for the fitting assessment, provides an estimation of the three Fréchet parameters and the work of fracture, as the normalizing fracture parameter. Further, the Young's modulus, as related to the ascendant part of the fracture curve, and the tension stress $\mathrm{f}_{\mathrm{ct}}$, as related to the distribution mode, i.e., the peak of the fracture curve, could be also estimated.

\section{Steps in the experimental testing and recorded data}

Fitting the experimental results to a GEV fracture curve comprises the following steps:

Step 1. Recording the experimental fracture curve from the 3-PB test.

The whole $P-\delta$ fracture curve for the concrete studied is recorded along the fracture process from the test initiation up to specimen failure, building a vast experimental database of $P-\delta$ value pairs. In principle, the experimental program is focused on the 3-PB test. Sometimes, a previous filtering of the anomalous outgoing fracture curve record from the experimental results, exemplary in the post-peak region, are required; in particular sudden load up and down arises due to the random and irregular fracture of aggregates, see Fig. 1.

Step 2. Enhancing the quality of the $P-\delta$ curve by homogenizing weights of all recorded data along the fracture curve $P-\delta$.

Usually, the 3-PB test is performed by CMOD control with a clipgauge transducer, whereby the load and the vertical displacement at the mid-span section are recorded too. The vertical displacement is measured by using a LVDT transducer mounted on a rigid frame to avoid parasitic torsional effects.

This implies that higher density of registered points, as projected to abscissa axis, is obtained where fracture curve parts exhibits larger slope whereas lower density results where the test rate speeds up, i.e. where it trends to horizontality, This is particularly notable at the upper tail where small changes of the load magnitude correspond to very larger variations of the $\delta$ values, implying implicitly that more weight is assigned to the numerous number of registered points in the pre- and post-peak region than to those located on the upper tail.

As a result, unsatisfactory fitting of the fracture curve is observed in the upper tail region, see red line of Fig. 3, what consequently induces a wrong extrapolation beyond the test scope impeding the correct fracture energy estimation.

A way to compensate this undesirable effect consists in expurgating the results in the high density regions of the fracture curve so that a regular data spacing along the fracture curve, in this case $\Delta \delta=0.001 \mathrm{~mm}$, is achieved allowing a practically homogeneous weighted data sample to be considered in the fitting process of the registered $P-\delta$ fracture curve. A plot representing the number of points included in the fitting process applied to the three representative zones of the fracture curve as considered here, is given in Fig. 2 b). In this way, more emphasis is given to the tail point registered in the tail and the improved fitting achieved becomes apparent, see green dotted line of Fig. 3. Other alternative procedures, as assigning different weights according to the different slope region of the fracture curve could be also envisaged.

The operation loop implemented in the fitting Matlab software consists in:

- A displacement vector ranging from a minimum 0 value to the maximum experimental limit test displacement is created assuming regular increments of $0.001 \mathrm{~mm}$.

- For any displacement value of the displacement vector just created, the corresponding paired load value is found out from the experimental vector registered. This ensures pair of $P-\delta$ values at regular displacement increments.

- Just in case the precise displacement value needed to define the next searched pair of values $P-\delta$ is not found, a linear interpolation is performed between the pair of values before and after the required displacement allowing an estimation of the searched load to be achieved.

Step 3. Fitting the experimental fracture curve as a scaled probability distribution function (pdf).

In the following, the fracture curve is assumed to be identified as a scaled probability density function (pdf) pertaining to the family of a generalized extreme value distribution (GEVD), see [12]. In fact, a Fréchet distribution could be proposed directly as a fitting candidate although letting open the distribution type from the GEVD family enhances the objectivity of the procedure.

The final $P-\delta$ function becomes 

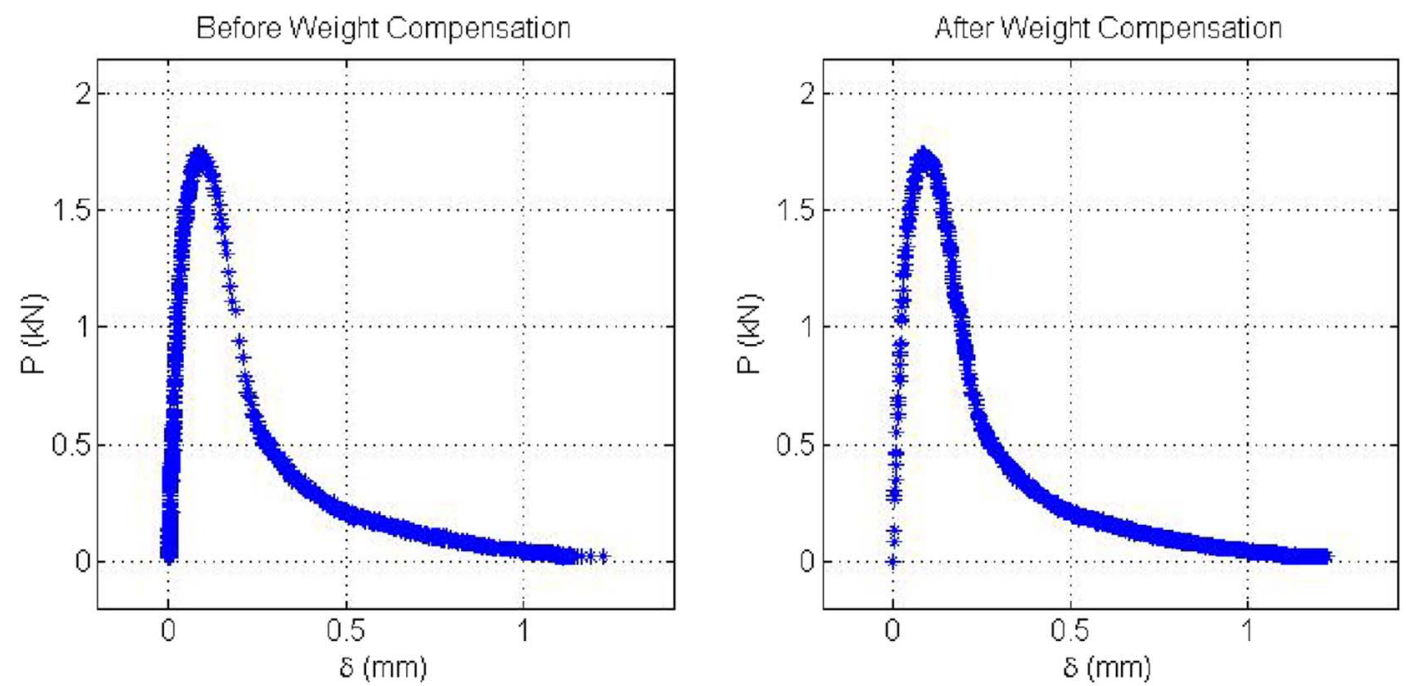

Fig. 2. Data density in experimental fracture curves: original data points as registered from the test (left) and data points used in curve fitting after weight compensation (right).

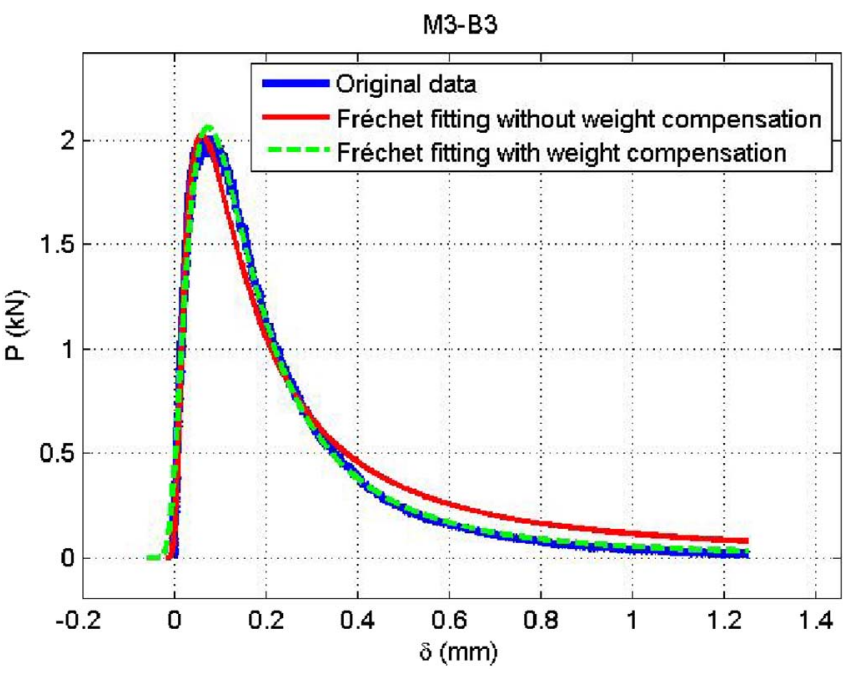

Fig. 3. Fitting of test record as delivered and after data weight compensation.

$P \delta=\Omega \cdot f_{G E V D}(\delta ; \lambda, \gamma, \kappa)$

where $f_{G E V D}(\lambda, \gamma, \kappa)$ is the GEVD characterized by $\lambda, \gamma, \kappa$, i.e. the location, scale and shape parameters, respectively:

$f_{G E V D}(\delta ; \lambda, \gamma, \kappa)=$

$$
\exp \left[-\left(1-\kappa\left(\frac{\delta-\lambda}{\gamma}\right)^{1 / \kappa}\right)\right] \cdot\left(1-\kappa \frac{\delta-\lambda}{\gamma}\right)^{\frac{1}{\kappa}-1} \cdot \frac{1}{\gamma}
$$

where $\delta \leq \lambda+\frac{\gamma}{\gamma}$, if $\kappa>0, \quad$ or $\delta \geq \lambda+\frac{\gamma}{\kappa}$, if $\kappa<0$ and $\Omega$ is a conversion factor denoted "fracture work scaling parameter" with work units that must be applied for the unit area under the pdf to equate the fracture work under the fracture curve. This ensures the model identification to be congruent from a dimensional point of view.

Step 4. Initial estimation of the GEVD parameters.

In order to speed up the convergence process by the parameter estimation procedure as well as to enhance the reliability of their values, a reasonable choice of the initial parameter values is advisable. A procedure providing those values independently of the particular $P-\delta$ studied would represent a desirable advance for the parameter estimation procedure. A rough estimation of the fracture work scaling parameter $\Omega$ is provided as the accumulated area under the fracture $P-\delta$ curve till the test limit, i.e. the measured fracture work, while the initial values of the remaining GEVD parameters $\lambda$, $\gamma$, and $\kappa$, are found based on the elemental percentile method proposed in [12] as a more suitable procedure than the maximum likelihood method. In this case the percentiles $0.1,0.3$ and 0.5 are considered in the calculation.

Step 5. Final estimation of the GEVD parameters.

With the aim of facilitating the final parameter estimation by an iteration process, a Matlab program is developed (see Annex 1 and 2), in which the initial values previously determined are introduced. It provides an optimal fitting of the analytical $P-\delta$ curve to the experimental results using the probability density function of a generalized extreme value (GEV) as already mentioned, see Expr. (2).

The parameters $\lambda, \delta$, $\kappa$, and $\Omega$ are determined by minimizing the difference between the curve defined by the function $P(\delta)=\Omega$. $f_{G E V D}(\delta ; \lambda, \gamma, \kappa)$ to be fitted and the original test results pairs using the least square method.

Step 6. Calculation of the work of fracture and fracture energy

The total fracture work cannot be determined directly from the test results due to data censoring caused by the limit displacement adopted in the real test. While the conventional assessment methods must resort to a complementary model to provide a prospective extrapolation of the fracture curve far upper tail, see [1,4-6], the present approach furnishes directly the fracture work as the fracture work scaling parameter $\Omega$. This happens because since $f_{G E V D}(\delta ; \lambda, \gamma, \kappa)$ is a pdf function, the integral of which from $\lambda$ to infinity is equal 1 , so that $\Omega$ can be identified as the integral of the $P-\delta$ curve from zero to infinity or equivalently as the total fracture work $W_{\text {total }}$ consumed in the test:

$\int_{0}^{\infty} P \delta d \delta=\int_{\lambda}^{\infty} \Omega \cdot f_{G E V D}(\delta ; \lambda, \gamma, \kappa) d \delta=\Omega \cdot \int_{\lambda}^{\infty} f_{G E V D}(\delta ; \lambda, \gamma, \kappa) d \delta=\Omega$

Further, the non-measured or remaining work of fracture, corresponding to the area under the far upper tail can be easily calculated from the analytical expression of the GEV pdf as its integral from the limit displacement in the experimental record, $\delta_{\text {lim }}$, up to infinity, giving us the opportunity to compare the values of such a remaining fracture work as given by the current estimation methods based on an extrapolation [1,4-6], and the GEVD approach proposed here.

Step 7. Identification of the particular resulting GEVD from the shape parameter value

Once the GEVD parameters are estimated, the sign of the shape parameter $\kappa$, determines the identification of the particular extreme value distribution, i.e. Weibull, Gumbel or Fréchet that fits optimally the experimental results. Without a unique exception in all the cases handled, the shape parameter $\kappa$ turns out to adopt a negative value confirming, without any supplementary assumption, that a Fréchet distribution for maxima is the searched solution. This allows the 
Table 1

Mix proportions of SCC mixes, $\mathrm{kg} / \mathrm{m}^{3}$.

\begin{tabular}{|c|c|c|c|c|c|c|c|c|c|c|c|}
\hline \multirow[t]{2}{*}{ Mix designation } & \multicolumn{3}{|l|}{$\mathrm{cm}^{\mathrm{a}}$} & \multirow[t]{2}{*}{ water } & \multirow[t]{2}{*}{$\mathrm{SP}^{\mathrm{b}}$} & \multirow[t]{2}{*}{$\mathrm{w} / \mathrm{cm}$} & \multirow[t]{2}{*}{$\mathrm{SP} / \mathrm{cm}(\%)$} & \multirow[t]{2}{*}{$\mathrm{LP}^{\mathrm{c}}$} & \multicolumn{2}{|l|}{$\mathrm{FA}^{\mathrm{d}}$} & \multirow[t]{2}{*}{$\mathrm{CA}^{\mathrm{e}}$} \\
\hline & cement & ggbs & $\mathrm{SF}$ & & & & & & $\mathrm{FA}^{\dagger}$ & $\mathrm{FA}^{\dagger \dagger}$ & \\
\hline M1 & 233 & 117 & - & 200 & 1.8 & 0.57 & 5 & 62 & 453 & 698 & 548 \\
\hline M2 & 207 & 152 & - & 200 & 1.8 & 0.56 & 5 & 58 & 426 & 709 & 540 \\
\hline M3 & 252 & 124 & - & 190 & 2.3 & 0.50 & 6 & 65 & 479 & 739 & 580 \\
\hline M4 & 405 & - & 196 & 144 & 20 & 0.24 & 34 & 48 & 352 & 434 & 796 \\
\hline M5 & 407 & - & 195 & 146 & 20 & 0.24 & 34 & 48 & 350 & 436 & 791 \\
\hline M6 & 429 & - & 137 & 131 & 16 & 0.23 & 28 & 54 & 396 & 691 & 560 \\
\hline
\end{tabular}

${ }^{\text {a }}$ Cementitious material, i.e. binder (Note: ggbs is the ground granulated blast furnace slag and SF refers to silica fume).

${ }^{b}$ Super-plasticizer (BASF Masterglenium SKY 886).

${ }^{\mathrm{c}}$ Limestone powder $<125 \mu \mathrm{m}$.

${ }^{\mathrm{d}}$ Fine aggregate $<2 \mathrm{~mm}$ (Note: a part of the fine aggregate is the coarser fraction of the limestone

powder, $\mathrm{FA}^{\dagger} 125 \mu \mathrm{m}-2 \mathrm{~mm}$, whereas $\mathrm{FA}^{\dagger \dagger}$ refers to natural river sand $<2 \mathrm{~mm}$ ).

e Coarse aggregate $<10 \mathrm{~mm}$

fracture curve to be optimally fitted irrespective of the concrete mix and strength, as expected from the considerations already advanced in Section 2.

The Fréchet distribution fulfils the condition of being a particular case of heavy tail distribution the pdf of which is given as

$f_{\text {Fréchet }}(\delta ; \lambda, \gamma, \beta)=$

$$
\frac{\beta}{\gamma}\left[\frac{\gamma}{\delta-\lambda}\right]^{\beta+1} \exp \left[-\left(\frac{\gamma}{\delta-\lambda}\right)^{\beta}\right] ; \quad \delta>\lambda
$$

$\lambda, \delta$ and $\beta$ being the location, scale and shape Fréchet parameters, respectively, to be obtained from the GEVD parameters, see (2), according to the expressions:

$\lambda=\lambda^{*}+\frac{\gamma^{*}}{\kappa^{*}}$

$\gamma=-\frac{\gamma^{*}}{\kappa^{*}}$

$\beta=-\frac{1}{\kappa^{*}}$

where the parameters marked with asterisk correspond to the GEVD function.

The same $P-\delta$ law as in (1) holds with the only change of the pdf being particularized to the Fréchet one:

$P \delta=\Omega \cdot f_{\text {Fréchet }}(\delta ; \lambda, \gamma, \kappa)$

Expr. (4) evidences that an extrapolation for the far upper tail of the fracture curve beyond the test limit should be performed as a product of an increasing exponential function times a decreasing potential function, a solution not at all evident to be foreseen in advance.

\section{Experimental program}

In this Section, the results of an extensive experimental program

Table 2

Flow test results of SCC mixes.

\begin{tabular}{lll}
\hline \multirow{2}{*}{ Mix designation } & \multicolumn{2}{l}{ Slump flow } \\
\cline { 2 - 3 } & $\begin{array}{l}\text { Spread } \\
\text { mm }\end{array}$ & $\mathrm{t}_{500}$ \\
& & $\mathrm{~s}$ \\
\hline M1 & 690 & 2.5 \\
M2 & 710 & 2.4 \\
M3 & 700 & 2.8 \\
M4 & 760 & 2.2 \\
M5 & 805 & 2.1 \\
M6 & 700 & 2.7 \\
\hline
\end{tabular}

carried out on 3-PBT specimens made of normal and high-strength selfcompacting concretes mixes with compressive strength ranging from 36 to $107 \mathrm{MPa}$ are presented. The three GEVD model parameters and the fracture work scaling parameter $\Omega$ are estimated from the test data, and the Fréchet pdf parameters and the total fracture work and energy are subsequently derived. In particular, the remaining non measured fracture work is calculated allowing a comparison to be made with the corresponding values as resulting from the model proposed by Elices et al. [4-6]. In this way, the applicability of the Fréchet distribution to fit the load-displacement curves can be checked.

\subsection{Materials}

The mixes were designed according to the method proposed by Deeb and Karihaloo [16]. The maximum aggregate size was $10 \mathrm{~mm}$ and the cement was an ordinary Portland cement CEMII/B-L $32.5 \mathrm{~N}$ for all mixes. Six different SCC mixes were manufactured with different volume fractions of coarse aggregates, paste to solid and water to binder ratios. The mix proportions and constituents are shown in Table 1. It should be noted that in the designation of the mixes the number corresponds to their target compressive strength. The specific gravity of the cement, ggbs and silica fume are 3.10, 3.30 and 2.25 respectively. In case of limestone, river sand and coarse aggregate is $1.70,2.65$ and 2.8 respectively. Table 2 shows the flow test results measured for the SCC mixes.

\subsection{Specimen preparation and test procedure}

From each of the six mixes (Table 1) 4 beam specimens $(100 \times 100 \times 440 \mathrm{~mm})$, four cubes $(100 \mathrm{~mm})$ and four cylinders $(100 \times 200 \mathrm{~mm})$ were cast. The mixes were prepared in a planetary mixer by mixing the coarsest constituent (coarse aggregate) and the finest one (micro-silica), followed by the next coarsest (sand) and next finest constituent (cement), and so on. Before each addition, the constituents were mixed for $2 \mathrm{~min}$. To fluidize the dry mix, two-thirds

Table 3

Results of mechanical properties and fracture parameters of SCC mixes.

\begin{tabular}{|c|c|c|c|}
\hline \multirow[t]{2}{*}{ Mix } & \multicolumn{3}{|c|}{ Mechanical properties } \\
\hline & $\begin{array}{l}f_{c} \\
(\mathrm{MPa})\end{array}$ & $\begin{array}{l}f_{s t} \\
(\mathrm{MPa})\end{array}$ & $\begin{array}{l}E_{c} \\
(\mathrm{GPa})\end{array}$ \\
\hline M1 & $34.4 \pm 2 \%$ & $3.2 \pm 3 \%$ & $30.7 \pm 1 \%$ \\
\hline M2 & $42.2 \pm 2 \%$ & $3.3 \pm 1 \%$ & $33.9 \pm 7 \%$ \\
\hline M3 & $51.4 \pm 2 \%$ & $4.0 \pm 9 \%$ & $35.1 \pm 6 \%$ \\
\hline M4 & $79.4 \pm 5 \%$ & $4.9 \pm 5 \%$ & $36.9 \pm 4 \%$ \\
\hline M5 & $96.8 \pm 4 \%$ & $5.3 \pm 5 \%$ & $42.3 \pm 1 \%$ \\
\hline M6 & $105.2 \pm 3 \%$ & $5.4 \pm 7 \%$ & $46.0 \pm 2 \%$ \\
\hline
\end{tabular}



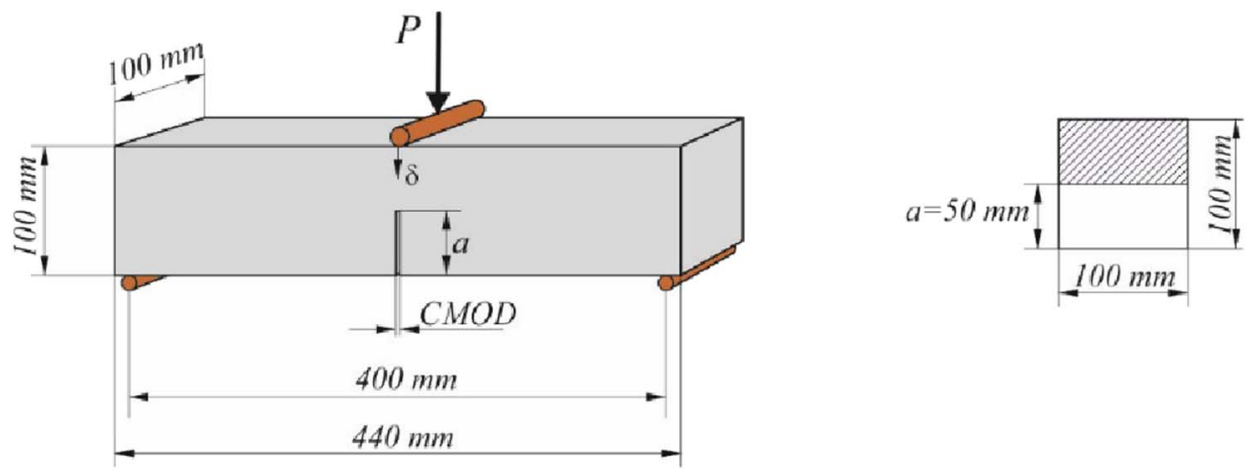

Fig. 4. Schematic representation of the 3-PB test configurations.

of the super-plasticiser (SP) was added to the water. One-half of this water-SP mixture was added to the dry constituents and mixed for $2 \mathrm{~min}$. One-half of the remaining water-SP mixture was then added and mixed for $2 \mathrm{~min}$. This process was continued until all water-SP mixture was added in about $10 \mathrm{~min}$. The remaining one-third of the SP was added and mixed for 2 min just before transferring the mix into the moulds. The specimens were de-moulded after 1 day and cured in water at ambient temperature for 28 days.

The characteristic compressive strength $\left(f_{c}\right)$ was determined from $100 \mathrm{~mm}$ cubes in accordance with UNE EN12930-3:2009. The indirect tensile strength $\left(f_{s t}\right)$ was obtained using the cylinder splitting test (Brazilian tests) according to UNE EN12930-6:2010, carried out on $100 \mathrm{~mm}$ diameter by $200 \mathrm{~mm}$ long cylinders. The static elastic modulus of concrete $\left(E_{c}\right)$ was determined according to the UNE EN12390-13:2014 by gradually loading a cylindrical specimen in compression to approximately a third of its failure load and measuring the corresponding strain from $30 \mathrm{~mm}$ strain gauges. The values of the mean and coefficient of variation measured for the mechanical properties of all concrete mixes are given in Table 3.

Regarding the fracture energy of SCC mixes, three-point bending tests were carried out on notched beams. The beams cast for each mix were notched to a depth of $50 \mathrm{~mm}$ (notch to depth ratio $a / W=0.5$ ) with a thin $(3 \mathrm{~mm})$ diamond saw. As schematically shown in Fig. 4, the tests for the determination of the fracture energy were performed according to the RILEM work-of-fracture method [7]. The crack mouth opening displacement (CMOD) was used as the feedback control signal and the load-point deflection was measured simultaneously by means of a linearly variable displacement transducer (LVDT) mounted on a rigid frame to avoid parasitic torsional effects on measurement of vertical displacement. The tests were performed in a stiff closed-loop universal testing machine with a maximum load capacity of $50 \mathrm{kN}$.

\subsection{Determination of the size-independent fracture energy according to the} method proposed by Elices et al.

The specific fracture energy of concrete is determined by means of the work-of-fracture method procedure of RILEM [7] with the corrections proposed by Guinea, Planas and Elices [4-6]. As is well known, the original RILEM procedure provides size-dependent values of the specific fracture energy of concrete. Guinea et al. studied this size dependency and attributed it to the presence of several sources of energy dissipation in the test procedure. Among others, the most important source is the non-measured specific fracture energy of concrete due to the curtailment of the tail part of the load-displacement curve $(P-\delta)$. It should be noted that other procedure could be used to determine the size-independent value of the specific fracture energy as the boundary effect method proposed by Hu, Wittmann and Duan [17-20] or its simplified version proposed by Abdalla and Karihaloo [10] and validated by Karihaloo et al. [21].

To estimate this non-measured work of fracture when the test is finished $\left(W_{F . N M}\right)$ at very low loads it is necessary to model the beam behavior when the cohesive crack closely approaches to the free surface [6]. For cohesive materials like concrete, the last phase of a stable three-point bending test can be modeled following the rigid-body kinematics used by Petersson [2].

Considering the geometrical relationships given by the rigid-body model, the non-measured fracture energy of the three-point bend test must be estimated by (7) according to [6]:

$W_{F . N M}=\frac{A}{\delta_{u}}$

where $A$ is the experimental coefficient for fitting the $P-\delta$ tail (also known as the far tail constant) and $\delta_{u}$ is the last recorded midspan deflection of the specimen at the end of the test.

Once the non-measured energy has been estimated, the sizeindependent fracture energy of concrete can be calculated from Expr. (8), see [22]:

$G_{F}=\frac{W_{F . M}+W_{F . N M}}{A_{\text {lig }}}=\frac{\int_{0}^{\delta_{u}} P d \delta}{B \cdot D-a}$

where $A_{\text {lig }}$ is the ligament area to be broken during the test.

As observed, the tail of the $P-\delta$ curve must be fitted between the two end points ( $\delta_{0}$ and $\delta_{u}$ ) to derive the coefficient $A$ (Fig. 5).

As demonstrated by Lee and Lopez [1], the estimated non-measured work of concrete is highly sensitive to the far tail constant value, which depends on the values of both end points $\left(\delta_{o}\right.$ and $\delta_{u}$ ) considered for fitting the tail. They found that the appropriate selection of the end points of the test should be considered along with the maximum aggregate size in order to obtain a consistent value of the sizeindependent fracture energy of concrete. Elices et al. [4-6] established that a general criterion consists in considering $\delta_{0}$ as the vertical displacement corresponding to a load of $5 \%$ of the peak load $\left(P_{\max }\right)$ and $\delta_{u}$ as the last recorded mid-span deflection of the specimen at the end of the test.

\section{Results}

Table 4 shows the results (mean values and COV) of the total work of fracture and the size-independent fracture energy for the different SCC mixes considering the correction to fit the tail of the $P-\delta$ curve. The results are similar to those found by other authors for similar strength SCC mixes [22-24]. It should be noted that results showed in Table 4 are those obtained for a value of $\delta_{0}$ corresponding to the $5 \%$ of the peak load. In Annex 3 the values of the different necessary fitting values of $\delta_{0}$ corresponding to a load of $7.5 \%, 5 \%$ and $2.5 \%$ of the peak load, respectively, are considered to analyze the variability of results for the $\delta_{o}$ chosen. $\Delta W_{F . N M-5 \%}$ represents the variation of the non-measured work of fracture estimated according to the recommended load of $5 \%$ of the peak load. As observed, the estimated value of $W_{F . N M}$ shows high relative variations with respect to the chosen $\delta_{0}$ in some cases. 


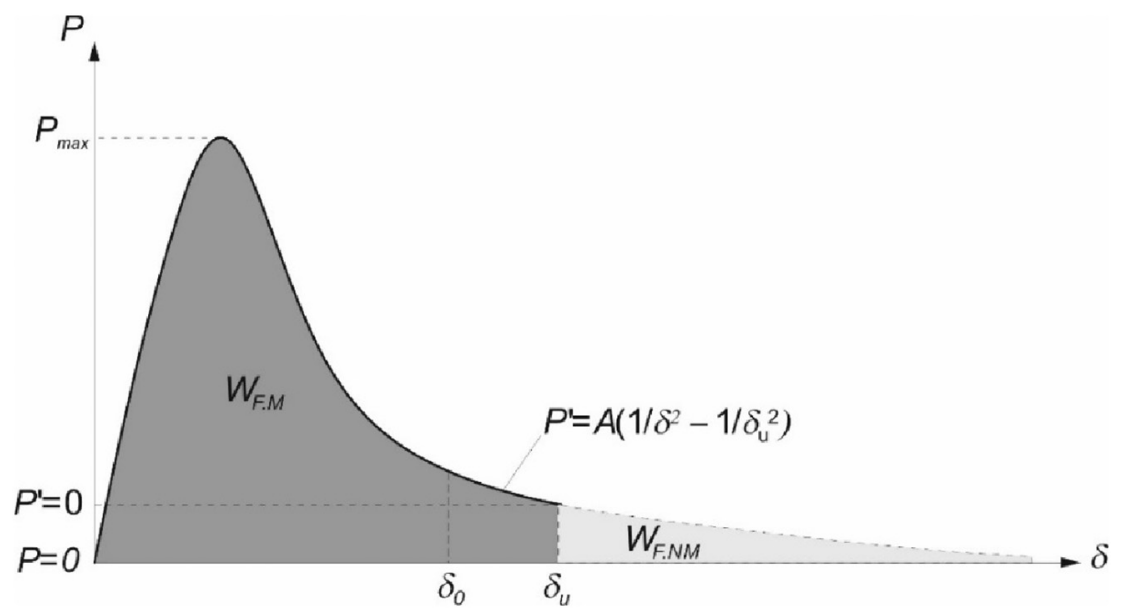

Fig. 5. $P-\delta$ curve in a three-point bend test and the measured $\left(\mathrm{W}_{\mathrm{F} . \mathrm{M}}\right)$ and non-measured $\left(\mathrm{W}_{\mathrm{F} . \mathrm{NM}}\right)$ work of fracture.

Table 4



\begin{tabular}{|c|c|c|c|c|c|c|c|c|}
\hline \multirow[t]{2}{*}{ Mix } & \multicolumn{4}{|l|}{ Elices et al. } & \multicolumn{4}{|l|}{ GEVD Model } \\
\hline & $\begin{array}{l}\mathrm{W}_{\mathrm{F} . \mathrm{M}} \\
(\mathrm{Nmm})\end{array}$ & $\begin{array}{l}\mathrm{W}_{\mathrm{F} . \mathrm{NM}} \\
(\mathrm{Nmm})\end{array}$ & $\begin{array}{l}\mathrm{W}_{\mathrm{F} . \mathrm{T}} \\
(\mathrm{Nmm})\end{array}$ & $\begin{array}{l}\mathrm{G}_{\mathrm{F}} \\
(\mathrm{N} / \mathrm{m})\end{array}$ & $\begin{array}{l}\text { W: }{ }_{\mathrm{F} . \mathrm{M}} \\
(\mathrm{Nmm})\end{array}$ & $\begin{array}{l}\text { W:*.N M } \\
(\mathrm{Nmm})\end{array}$ & $\begin{array}{l}\text { WF.T } \\
(\mathrm{Nmm})\end{array}$ & $\begin{array}{l}\mathrm{G}_{\mathrm{F}}^{*} \\
(\mathrm{~N} / \mathrm{m})\end{array}$ \\
\hline M1 & $536 \pm 13 \%$ & $87 \pm 46 \%$ & $623 \pm 17 \%$ & $125 \pm 17 \%$ & $543 \pm 14 \%$ & $73 \pm 32 \%$ & $616 \pm 16 \%$ & $123 \pm 16 \%$ \\
\hline M2 & $490 \pm 15 \%$ & $62 \pm 23 \%$ & $553 \pm 16 \%$ & $111 \pm 16 \%$ & $491 \pm 16 \%$ & $50 \pm 51 \%$ & $541 \pm 19 \%$ & $108 \pm 19 \%$ \\
\hline M3 & $528 \pm 10 \%$ & $68 \pm 20 \%$ & $597 \pm 10 \%$ & $119 \pm 10 \%$ & $526 \pm 11 \%$ & $34 \pm 56 \%$ & $560 \pm 13 \%$ & $112 \pm 13 \%$ \\
\hline M4 & $393 \pm 11 \%$ & $50 \pm 22 \%$ & $443 \pm 12 \%$ & $89 \pm 8 \%$ & $399 \pm 12 \%$ & $22 \pm 52 \%$ & $421 \pm 13 \%$ & $84 \pm 13 \%$ \\
\hline M5 & $428 \pm 15 \%$ & $71 \pm 35 \%$ & $499 \pm 17 \%$ & $100 \pm 17 \%$ & $428 \pm 15 \%$ & $28 \pm 36 \%$ & $455 \pm 15 \%$ & $91 \pm 15 \%$ \\
\hline M6 & $386 \pm 16 \%$ & $46 \pm 43 \%$ & $432 \pm 18 \%$ & $86 \pm 18 \%$ & $394 \pm 17 \%$ & $18 \pm 54 \%$ & $412 \pm 19 \%$ & $82 \pm 19 \%$ \\
\hline
\end{tabular}

The fracture curves from the experimental program are also fitted using the GEVD model with the aid of the Matlab code given in Annex 1. Captures of the Matlab program prepared as User's Guide are also given in Annex 2. The mean values and COV of the work of fracture and size-independent specific fracture energy calculated with the proposed method are also provided in Table 4 so that, subsequently, the values from both methods can be easily compared. In Annex 4, the corre- sponding GEVD model parameters obtained for each of the analyzed beams are exposed giving advice of the quality of the fitting reached.

Fig. 6 shows a selection of fitted density functions for the different mixes plotted along with the experimental recorded data. For the sake of simplicity, only one of the fracture curves for each sample, i.e. concrete mix, is displayed. The GEVD fit can be judged as highly satisfactory. Only in three cases (from a total amount of twenty three


Fig. 6. Original fracture curves from recorded data and fitted GEVD function representative of each concrete mix M1 to M6. 


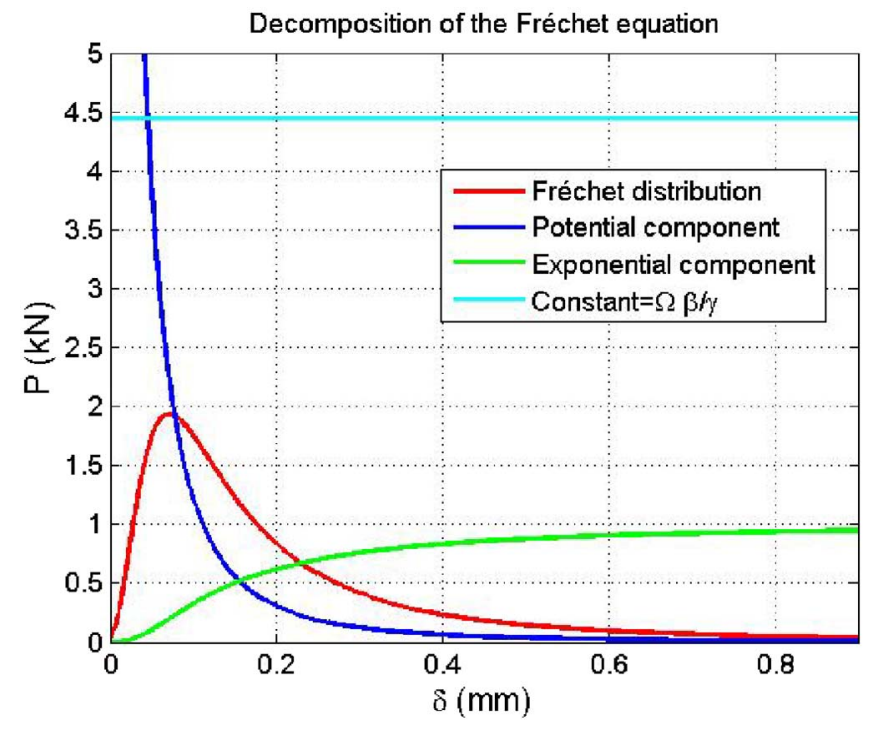

Fig. 7. Graphical representation of the two potential (blue) and exponential (green) components, constituting the Fréchet density function, see (4). (For interpretation of the references to color in this figure legend, the reader is referred to the web version of this article.)

beams tested) a slight feet would be advisable.

It should be noted that the GEVD approach provides directly the total fracture work identifiable as the value of the $\Omega$ parameter. In order to compare the results of the fracture energy obtained using the GEVD approach with those issuing from the method proposed by Elices et al. [4-6], the partial work of fracture, as resulting from the area under the fracture curve measured from the test record, and the non-measured work of fracture, are also included in Table 4. The values calculated with the GEVD model are those corresponding to the columns marked with an asterisk.

\section{Discussion}

The results of the measured fracture work, $W_{F . M}$, corresponding to the area under the fracture curve up to the test limit, are in good agreement either using directly the experimental recorded results or the GEVD approach. On the contrary, substantial differences arise between the non-measured fracture work, $W_{F . N M}$, as estimated according to the GEVD approach and as proposed by Elices et al. [4-6], and recommended in [7]. Higher confidence can be assigned to the former since its results are supported by the throughout fitted fracture curve, which is not influenced by the choice of the limiting displacement, $\delta_{0}$, contrary to the results provided by the latter, as pointed out by Lee and Lopez [1]. According to the assessment of the results for the five concrete mixes, it can be concluded that the non-measured fracture work results estimated by the proposal of Elices et al. for $\delta_{0}\left(5 \% \mathrm{P}_{\max }\right)$ surpasses systematically those provided by the present approach. This implies unsafe overestimation of the fracture energy of the concrete. This could be explained as a consequence of assuming a solution of exponential type for the extrapolation curve instead of the correct one, represented presumably by the Fréchet density function, which represents the product of a potential type component times an exponential one, the latter, by the way, not approaching asymptotically to zero but increasing to unity (see Fig. 7). The GEVD solution, as a particular case of heavy tail distribution, approaches closer to the displacement axis than the pure exponential solution thus providing less area under the far tail of the curve than that as presumed by the solution of Elices et al. [4-6].

Since the maximum aggregate size is maintained constant throughout all concrete mixes, the variation exhibited by the model parameters may be expectedly attributed to the mechanical properties of the mix, for instance to the compressive strength, as long as a systematic parameter investigation is not undertaken. Accordingly, apart from some small inconsistencies possibly due to the limited number of the specimens included in the six concrete mix samples tested, a clearly decreasing trend of the fracture curve scaling parameter $\Omega$ is noticed, while a significant increasing trend is exhibited by the Fréchet shape parameter for increasing values of the compressive strength. The Fréchet scale parameter seems to be insensible to the concrete strength, at least when the maximum aggregate remains unaltered. On its turn, the absolute variation of the Fréchet location parameter is not significant.

It should be noted that the Fréchet location parameter generally exhibits small but not nil values. This would point out that the transmission of the load from the machine to the specimen is not so simple and immediate as usually is taken for granted. In fact, an initial process is required before the linear ascending pre-pick branch of the fracture curve occurs. In any case, the influence of this initial part of the curve on the fracture work is negligible.

The analytical expression of the fracture curve provided by the model proposed opens new perspectives for deriving the softening curve to be subsequently implied in FE calculations based on a cohesive model but also for exploring the possible use and standardization of alternative testing specimens to determine fracture energy, as for instance the so-called "modified disc compact test" (MDCT), proposed by the authors [23], which proves to represent a reliable and simple test, particularly suitable also for assessing fracture properties of already casted concrete structures.

Finally, it must be insisted on the statistical grounds on which the model herein proposed is based, which strengthen the real statistical character of the fracture process in concrete. As stated by Hu et al. in a recent paper [8], the coarse aggregate structure of concrete has a crucial role on the peak load and the softening branch, after the peak, of the $P-\delta$ curve and subsequently, on fracture parameters of concrete, such as the tensile strength, the specific fracture energy and size effect. It is well known the influence of the internal structure of concrete on its fracture behavior and subsequently, of its mix proportioning, as demonstrated by several researchers [24-26], especially in SCC where a higher amount of fine particles is necessary [27]. An increase of the CA fraction produces an increase of $G_{F}[24,28]$, originated by the greater softening part of the $P-\delta$ curve. Moreover, as demonstrated by Alyhya et al. [24] an increase in the $p / s$ ratio provides a decrease of the fracture energy, as well as the reduction of $G_{F}$ with decreasing the $w / \mathrm{cm}$ ratio [24-26]. As observed from results (Table 4), the mixes herein studied follow the same trends depending on its mix proportions (Table 1). The presence of the coarse aggregate structure implies a remarkable heterogeneity of the concrete, which combined with the random distribution of the coarse aggregates in the matrix and its influence on the shape of the real crack front reveals the mechanisms acting behind the statistical nature of the fracture process in quasibrittle materials, as considered in the Hu-Duan boundary effect model [8]. It is expected that the analytical description of the fracture process would contribute to help in clarifying the conceptual discussion concerning the Bazant size effect model (SEM) and the BEM, see $[8,29-31]$. It will be of interest to investigate in a next experimental research program the influence of the coarse aggregate structure (distribution and maximum size) on the Fréchet parameters, particularly the scale and shape ones. The same applies for the specimen size.

\section{Conclusions}

The main conclusions that can be drawn from this work are the following:

- The fracture process of quasi brittle materials, in particular that of concrete, is supposed to represent a phenomenon of statistical character that can be identified with a probability density function of the GEVD family as a particular case of heavy tail distributions. At least some of 
the parameters can be identified with physical magnitudes related to the fracture process.

- A Fréchet distribution for maxima is "a priori" justified on considerations based on extreme value statistics and confirmed as the general solution in all the cases studied. This expectation is confirmed since the GEVD probability density function fitting optimally the experimental $P-\delta$ data recorded during the fracture process to a fracture curve turns out to be regularly a Fréchet for maxima. This supports the assumption of the statistical character of the micromechanical damage of the material during the real fracture process.

- The GEVD model is applied to 3-PB test records from an experimental program carried out on an ample variety of concrete mixes. The Fréchet density function for maxima provides repeatedly high satisfactory fitting throughout the $P$ - $\delta$ curve proving to represent a robust proposal for fitting the fracture curve and estimating the fracture energy of concrete, independently of the concrete mix considered.

- The results of the measured fracture work, $W_{F . M}$, provided by the GEVD approach are in good agreement with those derived directly from the test recorded results whereas the non-measured fracture work, $W_{F . N M}$, as derived according to the method recommended by RILEM TCM-85 systematically exceeds that obtained applying the GEVD approach, to which incidentally higher confidence can be assigned since the fracture curve is in such a case fitted throughout the recorded test data.

- A Matlab program is developed for automatic determination of the model parameters. The program provide the fracture energy measured and non-measured, represented by the scale parameter according to the fitted GEVD distribution, the Fréchet parameters, the maximum load by the specimen identified as the distribution mode and an estimation of the tension strength for the concrete specimen tested.

\section{Acknowledgements}

The authors acknowledge partial support to this work supplied in the frame of the Research Project BIA2010-19920 of the Ministry of Science and Education and from Regional Project SV-PA-11-012 of the Chancellery of Science and Education of the Asturias Regional Government and Project BIA2016-75431-R of the Ministry of Economy and Competitiveness of Spain.

\section{Appendix A. Supplementary data}

Supplementary data to this article can be found online at http://dx. doi.org/10.1016/j.matdes.2017.05.030.

\section{References}

1] J. Lee, M.M. Lopez, An experimental study on fracture energy of plain concrete, Int. J. Concr. Struct. Mater. 8 (2014) 129-139, http://dx.doi.org/10.1007/s40069-0140068-1.

[2] P.E. Petersson, Fracture energy of concrete: method of determination, Cem. Concr. Res. 10 (1980) 78-89.

[3] RILEM, TC-89, Size-effect method for determining fracture energy and process zone size of concrete, Mater. Struct. 23 (1990) 461-465.

[4] G.V. Guinea, J. Planas, M. Elices, Measurement of the fracture energy using threepoint bend tests: part 1-influence of experimental procedures, Mater. Struct. 25 (1992) 212-218.

[5] J. Planas, M. Elices, G.V. Guinea, J. Planas, Measurement of the fracture energy using three-point bend tests: part 2 - influence of bulk energy dissipation, Mater. Struct. 25 (1992) 305-312.

[6] M. Elices, G.V. Guinea, J. Planas, Measurement of the fracture energy using three- point bend tests: part 3 - influence of cutting the P- $\delta$ tail, Mater. Struct. 25 (1992) 327-334.

[7] RILEM, TCM-85, Determination of the fracture energy of mortar and concrete by means of three-point bend tests on notched beams, Mater. Struct. 18 (1985) 287-290.

[8] X. Hu, J. Guan, Y. Wang, A. Keating, S. Yang, Comparison of boundary and size effect models based on new developments, Eng. Fract. Mech. 175 (2017) 146-167, http://dx.doi.org/10.1016/j.engfracmech.2017.02.005.

[9] B.L. Karihaloo, Fracture Mechanics and Structural Concrete, Longman Scientific and Technical Publishers, USA, 1995.

[10] H.M. Abdalla, B.L. Karihaloo, Determination of size-independent specific fracture energy of concrete from three-point bend and wedge splitting tests, Mag. Concr. Res. 55 (2003) 133-141.

[11] B.L. Karihaloo, A.R. Murthy, N.R. Iyer, B.L. Karihaloo, N.R. Iyer, B.K. Raghu Prasad, Determination of size-independent specific fracture energy of concrete mixes by the tri-linear model, Cem. Concr. Res. 49 (2013) 82-88, http://dx.doi.org/10.1016/j. cemconres.2013.03.010

[12] E. Castillo, A.S. Hadi, N. Balakrishnan, J.M. Sarabia, Extreme Value and Related Models with Applications in Engineering and Science, Wiley Seri, Hoboken, New Jersey, 2004.

[13] V. Pisarenko, M. Rodkin, Heavy-tailed distributions in disaster analysis, Ser. Adv. Nat. Technol. Hazards Res, 30 Springer, 2010.

[14] J.P. Nolan, Stable Distributions: Models for Heavy Tailed Data, Retrieved 2009-0221 (2009) (jpnolan@american.edu ).

[15] K. Sigman, Appendix: a primer on heavy-tailed distributions, Queueing Syst. Theory Appl. 33 (1/3) (1999) 261-275, http://dx.doi.org/10.1023/A:1019180230133.

[16] R. Deeb, B.L. Karihaloo, Mix proportioning of self-compacting normal and highstrength concretes, Mag. Concr. Res. 65 (2013) 546-556.

[17] X.Z. Hu, F.H. Wittmann, Fracture energy and fracture process zone, Mater. Struct. 25 (1992) 319-326.

[18] K. Duan, X. Hu, F. Wittmann, Size effect on specific fracture energy of concrete, Eng. Fract. Mech. 74 (2007) 87-96, http://dx.doi.org/10.1016/j.engfracmech. 2006.01.031.

[19] X. Hu, K. Duan, Mechanism behind the size effect phenomenon, J. Eng. Mech. 136 (2010) 60-68, http://dx.doi.org/10.1061//asce/em.1943-7889.0000070.

[20] X. Hu, K. Duan, Influence of fracture process zone height on fracture energy of concrete, Cem. Concr. Res. 34 (2004) 1321-1330, http://dx.doi.org/10.1016/j. cemconres.2003.12.027.

[21] B.L. Karihaloo, H.M. Abdalla, T. Imjai, A simple method for determining the true specific fracture energy of concrete, Mag. Concr. Res. 55 (2003) 471-481.

[22] H. Cifuentes, M. Alcalde, F. Medina, Measuring the size-independent fracture energy of concrete, Strain 49 (2013) 54-59.

[23] H. Cifuentes, M. Lozano, T. Holusova, F. Medina, S. Seitl, A. Fernandez-Canteli, Modified disk-shaped compact tension test for measuring concrete fracture properties, Int. J. Concr. Struct. Mater. (n.d.). doi:http://dx.doi.org/10.1007/ s40069-017-0189-4.

[24] W.S. Alyhya, M.S. Abo Dhaheer, M.M. Al-Rubaye, B.L. Karihaloo, Influence of mix composition and strength on the fracture properties of self-compacting concrete, Constr. Build. Mater. 110 (2016) 312-322, http://dx.doi.org/10.1016/j. conbuildmat.2016.02.037.

[25] M. Karamloo, M. Mazloom, G. Payganeh, Influences of water to cement ratio on brittleness and fracture parameters of self-compacting lightweight concrete, Eng. Fract. Mech. 168 (2016) 227-241, http://dx.doi.org/10.1016/j.engfracmech.2016. 09.011.

[26] M.H.A. Beygi, M.T. Kazemi, I.M. Nikbin, J.V. Amiri, The effect of water to cement ratio on fracture parameters and brittleness of self-compacting concrete, Mater. Des. 50 (2013) 267-276, http://dx.doi.org/10.1016/j.matdes.2013.02.018.

[27] H. Cifuentes, B.L. Karihaloo, Determination of size-independent specific fracture energy of normal- and high-strength self-compacting concrete from wedge splitting tests, Constr. Build. Mater. 48 (2013) 548-553.

[28] M.H.A. Beygi, M.T. Kazemi, I.M. Nikbin, J. Vaseghi Amiri, S. Rabbanifar, E. Rahmani, The influence of coarse aggregate size and volume on the fracture behavior and brittleness of self-compacting concrete, Cem. Concr. Res. 66 (2014) 75-90, http://dx.doi.org/10.1016/j.cemconres.2014.06.008.

[29] J. Guan, X. Hu, Q. Li, In-depth analysis of notched 3-p-b concrete fracture, Eng. Fract. Mech. 165 (2016) 57-71, http://dx.doi.org/10.1016/j.engfracmech.2016.08 020.

[30] Y. Wang, X. Hu, L. Liang, W. Zhu, Determination of tensile strength and fracture toughness of concrete using notched 3-p-b specimens, Eng. Fract. Mech. 160 (2016) 67-77, http://dx.doi.org/10.1016/j.engfracmech.2016.03.036.

[31] Z.P. Bažant, C.G. Hoover, Comparison of the Hu-Duan boundary effect model with the size-shape effect law for quasi-brittle fracture based on new comprehensive fracture tests, J. Eng. Mech. 140 (2014) 480-486, http://dx.doi.org/10.1061/ (ASCE)EM.1943-7889.0000632. 